INTERNATIONAL DESIGN CONFERENCE - DESIGN 2018

https://doi.org/10.21278/idc.2018.0549

\title{
THE INFLUENCE OF MATERIAL PROPERTY VARIANCES AND PRINTING TOLERANCES ON THE MECHANICAL BEHAVIOR OF AN ADDITIVELY MANUFACTURED META- MATERIAL TANK TRACK BACKER PAD
}

\author{
S. Franklin, G. Fadel, G. Li and N. Coutris
}

\begin{abstract}
This paper explores the influences that potential variances in material properties and nominal dimensions have on the overall mechanical behavior of an additively manufactured meta-material. The investigation looks at deviations between expected and experimental mechanical responses obtained through performance validation testing. Three sources for discrepancies were identified through literature review and model/experimental comparison. Sensitivity analyses were employed to obtain the significance of the design parameters, and preliminary work in boundary condition improvements is discussed.
\end{abstract}

Keywords: additive manufacturing, simulation based design, experimentation, sensitivity analysis, metamaterial

\section{Introduction}

Over the last several decades, additive manufacturing (AM) has provided an avenue for significantly expanded exploration of the mechanical design space. The layered building approach of the additive process provides unique advantages for designers, allowing for non-traditional geometries and previously unavailable weight reduction techniques, such as fully or partially enclosed voids, to be incorporated into the reservoir of design techniques. These newly unlocked geometries and topologies have also expanded the powers of optimization tools, and the feasibility of the designs they generate.

Utilizing the advantages of AM, meta-material design has become a topic of extreme interest in the performance and material design realms. The term meta-material has been defined by Cui et al. (2010) as "a macroscopic composite of periodic or non-periodic structure, whose function is due to both cellular architecture and the chemical compositions", meaning they rely as much on the benefits of cellular structure as they do on their constitutive material properties. These materials can be applied to a wide range of challenges, such as the development of high strength, light weight structures (Wang, 2005; Rosen et al., 2006), the absorption of crushing and impact energies (Sun et al., 2010; Schultz et al., 2012), non-pneumatic applications with high shear flexure and strains (Ju et al., 2010; Thyagaraja et al., 2011), maximizing heat transfer and dissipation (Gu et al., 2001; Wadley and Queheillalt, 2007), acoustic absorption and vibrational damping (Ruzzene, 2003; Varanasi et al., 2013), and several others. 
But while models may predict favorable performance and properties for these cellular materials, real mechanical behavior needs to be validated, specifically when additive technologies are being utilized. Many different methods currently exist for the production of metal powder based AM parts. With each one of those methods come expected variations and known tolerances that can be reflected in the final product. Material properties are known to be directly affected by process parameters such as powder layer thickness (Safdar et al., 2012) and porosity related to powder density (Levy et al., 2012), which can leave voids of unsolidified powder within the interior of parts. Dimensional inaccuracies can be the result of any number of factors, such as curved features, the g-code obtained from the part file, and the number of small features (Hernandez, 2015). Print orientation has also been identified as an important factor for both material properties and dimensional accuracy (Farzadi et al., 2014; Alharbi et al., 2016).

Further, research investigating the contributing factors of consistent and predictable additive manufacturing has been conducted (Spierings et al., 2011; Schade et al., 2014; Spierings et al., 2015; Strondl et al., 2015), but experimental data will help identify future work aimed at obtaining agreement between theoretical and physical designs. In conjunction, causes for the potential divergence between expected and real results must be identified and explored. This will allow for modifications to be made to related design processes and for an increase of design robustness and reliability. With that idea in mind, the driving goal of this particular paper is twofold. Validation of the "Modified Unit Cell Synthesis Method" (Kulkarni, 2016) is sought by analyzing part performance during experimental testing, while a sensitivity analysis is employed to ascertain the realistic range of response that can be expected from this design when manufactured under known tolerances. The notable deviations from the expected behavior are explored using both material and dimensional sensitivity analyses.

The paper is organized as follows: Section 2 covers background information on the "Modified Unit Cell Synthesis Method" for nonlinear meta-material design and a subsequent case study. A review of the compressive testing conducted on the resultant design is also covered. Section 3 discusses deviations experienced between the numerical and experimental results and identifies a set of factors that may contribute to the discrepancies. Section 4 presents the sensitivity analysis of these factors on the mechanical behavior of the pad. Finally, Section 5 provides conclusions and future work.

\section{Background}

\subsection{Unit Cell Synthesis Method}

The Unit Cell Synthesis Method is a meta-material design process presented by Satterfield et al. (2017) and modified by Kulkarni (2016), which is intended to generate cellular based materials that exhibit nonlinear deformation under loading conditions. The method utilizes functional beam elements, arranged in either a series or parallel orientation, much like electrical elements or springs, to obtain general target responses. Once an arrangement of beam elements and necessary structural pieces are deemed feasible, dimensional shape optimization is carried out, aimed at fine-tuning the response of the material to better match the desired mechanical behavior. A step by step representation of the modified method can be seen in Figure 1.

The synthesis method starts with a repository of essential functional geometries (EFG), which are represented by straight and curved beam elements. From this repository, combinations of elements are chosen, to best match the general behavior required by the global material. These combinations utilize the beam members in series, parallel, or both in order to best achieve the desired nonlinearity. Upon obtaining an appropriate general response, essential structural geometries (ESG) are added to the EFG configuration to form a complete unit cell, suitable for tessellation. A general design of experiments is then conducted on the meta-material, to determine if performance matching through optimization is a feasible proposition. If yes, then the material is subjected to a multi-objective optimization to confirm this feasibility and obtain the optimized dimensional parameters. If feasibility is determined to be no, at concept evaluation or after optimization, then the process returns to EFG selection and a new design is chosen. 


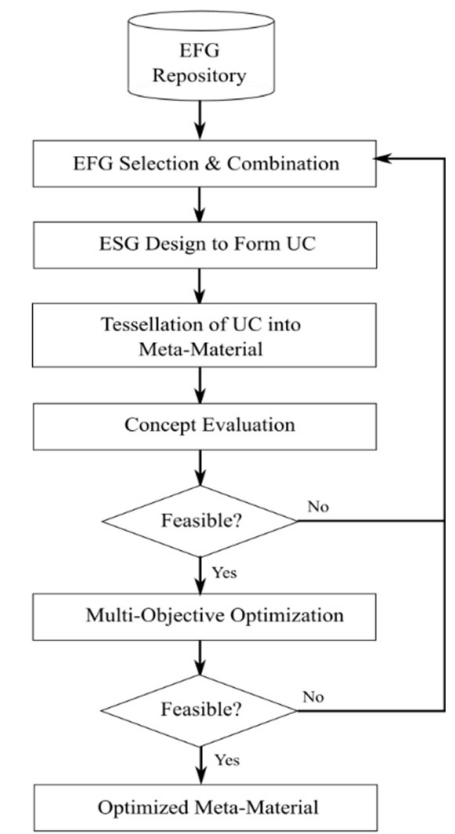

Figure 1. Modified Unit Cell Synthesis Method

In a subsequent case study, the method was employed to develop a meta-material pad aimed at replacing the elastomer backer pads on the track of the M1 Abrams tank. The chief objective of the design was to match the nonlinear behavior of the rubber pad while using a linear elastic bulk material that would not deteriorate due to hysteresis. A feasible design using a combination of cantilever and oval beams in series was chosen for the UC design, which can be seen in Figure 2. The pad was successfully optimized to minimize the strain error between its own response and the target response. Several pads were then manufactured for experimental testing and method validation, using one of the optimal set of design parameters along the Pareto-line. The physical pads were printed using an Arcam machine, which utilizes an electron-beam melting (EBM) technique to selectively melt and bond metal powder in accordance with the corresponding part layer geometry. The pads themselves were printed with Ti-6Al-4V, which is a grade 5 titanium alloy commonly used in metal AM fabrication of parts for aerospace and biomedical applications, due to its high strength and ability to withstand corrosion and high temperatures. Figure 3 shows a rendering of the manufactured pad, with the tessellated unit cell (UC) pattern.

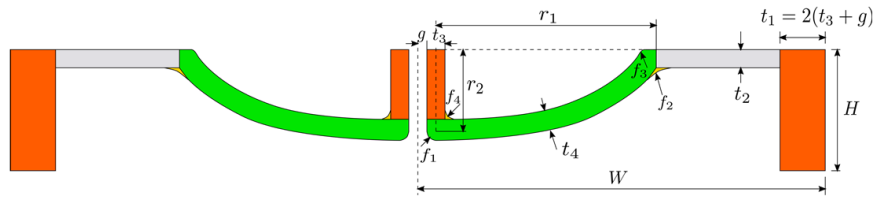

Figure 2. Canti-oval unit cell with identified variables and fillet positions

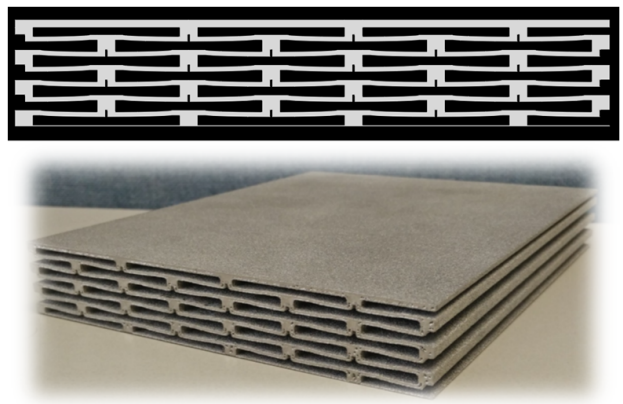

Figure 3. Tessellated unit cell pattern and 3D printed pad 


\subsection{Quasi-static compression testing}

In the original FE simulation, the maximum pressure applied was around 4MPa, which is on the order of $90 \mathrm{kN}$ of load. The maximum static load of the tank, spread across the 28 pads in contact at any given time, is $22.5 \mathrm{kN}$ or roughly $1 \mathrm{MPa}$ of pressure. Based off this new maximum, five near-uniform load increments, $5-10-15-20-22.5 \mathrm{kN}$, were selected and applied over a two-minute period, all starting from $0 \mathrm{kN}$. The displacements of the pad were recorded for each and the corresponding strains were calculated and plotted versus the applied pressure. In order to compare these strains against the expected FE model, the 5 same loads were used to obtain properly scaled simulation results. They were then plotted against the experimental results, which is shown in Figure 4. The first observation from the plot was the confirmation of nonlinear behavior by the experimental part, as the pad becomes stiffer with increased loading. However, there is a substantial difference in magnitude between the model and the experimental deformations. The physical pad exhibited nearly twice as soft of a response as the model predicted the deformations would be.

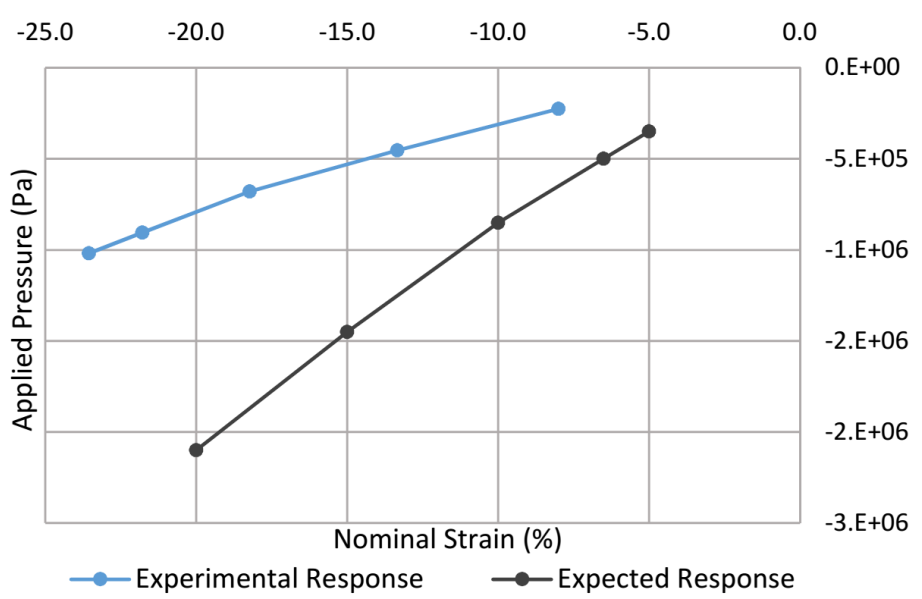

Figure 4. Comparison of expected and experimental pad results

\section{Investigation of model and experimental result discrepancies}

While discrepancies between the experiment and the numerical results are expected due to known variability from batch to batch or even part to part in additive manufacturing as discussed in Section 1, such a large deviation was quite puzzling. This problem prompted us to begin an investigation to determine causes for these discrepancies. However, a totally exhaustive investigation into these causes would be infeasible.

The focus of the investigation, therefore, turned to the consistency of the model inputs with regard to the physical part. That is to say, the software results are totally dependent on the inputs and therefore must accurately reflect the part that was tested, in order to obtain comparable results. This new direction was brought to light by information obtained from the manufacturers of the metamaterial pad. Chiefly, the tolerances of the printing process and the material properties of test specimens produced from the same powder batch as the pad. Since the pad was specifically optimized to minimize the strain error between the expected and desired deformations, errors in those dimensions would undoubtedly impact the overall performance. Similarly, differences in material stiffness from one to the other directly impact the behavior of the beam and support elements while loaded. This approach would also be significantly easier to explore and offers the possibility to tweak the FE model based on those property and dimensional analyses. As a result of this narrowed direction of the investigation, we were able to determine three potentially influential factors that could explain a large portion of the difference between the two mechanical responses. They are as follows:

1. The assigned material properties in the model, which were provided by the manufacturer, may not reflect the material properties of the built actual pad.

2. The printing tolerances of the EBM process may result in dimensional variances between the model and the physical part. 
3. The loading and boundary conditions of the model may not be indicative of the experimental conditions experienced by the physical pad.

Unfortunately, due to nature of the design, the limited number of pads that were initially printed, and the need to conduct future fatigue testing, destructive measures could not be taken to pinpoint dimensional errors and expensive scanning would take up time and resources needed to continue with the project. Additionally, when independently testing the material properties of a part, specimens need to be printed simultaneously using the same batch of powder and the same processing conditions. The material properties of metal powder can vary from batch to batch, and by the time compressive testing had been conducted and the need for independent property verification was deemed necessary, the manufacturer was printing with a newer batch of powder. This led us to further narrow our scope and look specifically at how potential errors in dimensions, changes in material properties, and adjustments for accurate experimental representation may account for the divergence in responses through a design of experiments and sensitivity analysis conducted using the model.

\section{Exploring potential sources of experimental/model deviation}

\subsection{Assigned material properties}

Early in the design process, performance requirements dictated that an elastic material with a relatively low ratio of Young's modulus to yield strength was needed. For this reason, titanium was selected as the pads base material. A Beta-C alloy was initially chosen to represent the pad material in the model, which exists at the low end of the stiffness spectrum for Ti alloys, at 102GPa. However, the EBM process used to manufacture the pads utilizes Ti-6Al-4V; a grade 5 alloy with Young's modulus range of 102-125GPa. To narrow the range, the manufacturer built tensile specimens for testing material properties, which were subsequently provided to us. The testing of these specimens showed the expected Young's modulus of our parts to be $\approx 114 \mathrm{GPa}$. This provided us with our first opportunity to update the original model and provide a more appropriate target response. With the new value of $E$ being stiffer than the original material selection, we expected an undesirable stiffening of the FE model but also anticipated the results would provide important information regarding the individual influence of material stiffness on the system.

In an effort to further our understanding of that influence and to explore the entire range of possible $E$ values, the response at $125 \mathrm{GPa}$ was also calculated via the model. A plot of the strains vs. stresses for the three moduli values can be seen in Figure 5.

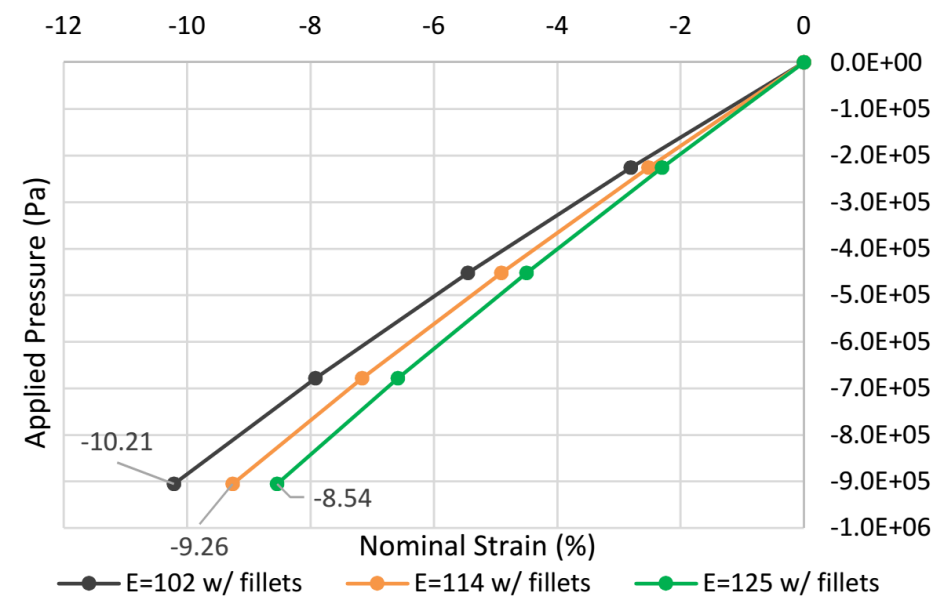

Figure 5. Modeled pad response at varying Young's modulus

These results show that as the modulus is decreased from $125 \mathrm{GPa}$ to $102 \mathrm{GPa}$, displacements increase in a nonlinear fashion. This is significant because, in linear elastic materials, increases or decreases in $\mathrm{E}$ result in inversely proportional changes to displacements, seen by the equation $\sigma / E=\varepsilon$, where $\sigma$ is the stress and $\varepsilon$ is the strain. Additionally, the change in $E$ from $102 \mathrm{GPa}$ to $125 \mathrm{GPa}$ resulted in a $16 \%$ change in final strain, which confirms the significant effect that variances in Young's moduli have on the overall 
performance. The emergence of a nonlinear trend between strains and Young's modulus, as well as the observed significance of a $10 \%$ change in material stiffness, could help explain a portion of the divergence between expected and experimental strain. It is important to also recognize that a high quality is being assumed for the print, since build material and process characteristics can cause print defects and sub-optimal material properties.

\subsection{Printing accuracy}

As a result of the optimization, the dimensional pad parameters were specifically tailored to match the response of the original rubber backers. In an ideal situation, the resulting printed parts would be an exact representation of the optimized model, to ensure consistency for the experimental testing. However, limitations in printing technologies do not allow for that level of accuracy. In fact, it is documented across many AM processes that inaccuracies among dimensions, which are sometimes quite significant, exist and need to be addressed (Dimitrov et al., 2006; Salmi et al., 2013; Lieneke et al., 2015). Additionally, work by Cooke and Soons (2010) shows that the EBM and other laser-based processes can incur dimensional inaccuracies a magnitude greater or more than a CNC machined counterpart. In one experiment, mean dimensional errors greater than $0.1 \mathrm{~mm}$ were observed for EBM parts. Further, work by Smith et al. (2016) shows that the EBM process tends to manufacture undersized members in truss structures, with a mass error shown to be greater than $30 \%$ in particular cases. It is, therefore, reasonable to expect the presence of variances between the optimized and physical pads, which would certainly help explain performance discrepancies.

\subsubsection{Design of experiment}

In an effort to capture the potential effects of these known printing tolerances, a sensitivity analysis was conducted, which took into account the 15 independent variables of the UC, as well as the top and bottom sheets, which are listed in Table 1 .

Table 1. Independent variables of printed pad

\begin{tabular}{|c|c|c|}
\hline Variable & Description & Optimized Value \\
\hline t2 & Cantilever Beam Thickness & $1.17 \mathrm{~mm}$ \\
\hline $\mathbf{t 3}$ & ESG Thickness & $1.84 \mathrm{~mm}$ \\
\hline t4 & Oval Beam Thickness & $1.11 \mathrm{~mm}$ \\
\hline $\mathbf{r 1}$ & Oval Beam Major Radius & $14.6 \mathrm{~mm}$ \\
\hline $\mathbf{r 2}$ & Oval Beam Minor Radius & $0.4 \mathrm{~mm}$ \\
\hline $\mathbf{g}$ & Gap & $0.2 \mathrm{~mm}$ \\
\hline $\mathbf{W}$ & Half Width of Unit Cell & $20.5 \mathrm{~mm}$ \\
\hline $\mathbf{H}$ & Height of Unit Cell & $3.2 \mathrm{~mm}$ \\
\hline $\mathbf{E}$ & Young's Modulus & $114 \mathrm{GPa}(\mathrm{avg} \mathrm{value})$ \\
\hline TT & Top Sheet Thickness & $1.7 \mathrm{~mm}$ \\
\hline BT & Bottom Sheet Thickness & $0.3 \mathrm{~mm}$ \\
\hline $\mathbf{f 1}$ & Fillet 1 Radius & $1.0 \mathrm{~mm}$ \\
\hline $\mathbf{f 2}$ & Fillet 2 Radius & $0.75 \mathrm{~mm}$ \\
\hline $\mathbf{f 3}$ & Fillet 3 Radius & $0.3 \mathrm{~mm}$ \\
\hline $\mathbf{f 4}$ & Fillet 4 Radius & $0.55 \mathrm{~mm}$ \\
\hline
\end{tabular}

For the analysis, the $\pm 0.1 \mathrm{~mm}$ printing accuracy of the EBM process was used as the two levels of a bi-level $\mathrm{L}_{16}\left(2_{15}\right)$ orthogonal array, which is a design of experiments that utilizes 15 variables in a 16 experiment setup, to determine the global effect of individual parameters on a system. In this case, the array was constructed using the 14 geometric variables and the one material property previously discussed. In this particular analysis, the 16 experiments were 16 individual models, generated using the corresponding high 
and low values that were obtained by adding or subtracting the accuracy from the original optimized parameter. Predefined sequences for $\mathrm{L}_{16}\left(2{ }_{15}\right)$ arrays, which are shown in Table 2, determine the particular combination of high and lows for any given experiment. They are specifically created to efficiently explore the design space without needing a fully exhaustive analysis. Also, to put the effects of the dimensional values in perspective with the effect of Young's modulus, the high and low values of the Ti-6Al-4V stiffness range were used for the corresponding high and low of the experiments. Once the 16 adjusted models were generated and run through the FE software, the strains were extracted and the individual effect coefficients were calculated. The equations used to find those values are seen below, while the individual parameter coefficients at each load and the combined average are shown in Table 3.

Table 2. $\mathrm{L}_{16}\left(2^{15}\right)$ orthogonal array table

\begin{tabular}{|c|c|c|c|c|c|c|c|c|c|c|c|c|c|c|c|}
\hline & A & B & C & D & E & F & G & H & I & J & K & L & M & N & O \\
\hline 1 & 1 & 1 & 1 & 1 & 1 & 1 & 1 & 1 & 1 & 1 & 1 & 1 & 1 & 1 & 1 \\
\hline 2 & 1 & 1 & 1 & 1 & 1 & 1 & 1 & 2 & 2 & 2 & 2 & 2 & 2 & 2 & 2 \\
\hline 3 & 1 & 1 & 1 & 2 & 2 & 2 & 2 & 1 & 1 & 1 & 1 & 2 & 2 & 2 & 2 \\
\hline 4 & 1 & 1 & 1 & 2 & 2 & 2 & 2 & 2 & 2 & 2 & 2 & 1 & 1 & 1 & 1 \\
\hline 5 & 1 & 2 & 2 & 1 & 1 & 2 & 2 & 1 & 1 & 2 & 2 & 1 & 1 & 2 & 2 \\
\hline 6 & 1 & 2 & 2 & 1 & 1 & 2 & 2 & 2 & 2 & 1 & 1 & 2 & 2 & 1 & 1 \\
\hline 7 & 1 & 2 & 2 & 2 & 2 & 1 & 1 & 1 & 1 & 2 & 2 & 2 & 2 & 1 & 1 \\
\hline 8 & 1 & 2 & 2 & 2 & 2 & 1 & 1 & 2 & 2 & 1 & 1 & 1 & 1 & 2 & 2 \\
\hline 9 & 2 & 1 & 2 & 1 & 2 & 1 & 2 & 1 & 2 & 1 & 2 & 1 & 2 & 1 & 2 \\
\hline 10 & 2 & 1 & 2 & 1 & 2 & 1 & 2 & 2 & 1 & 2 & 1 & 2 & 1 & 2 & 1 \\
\hline 11 & 2 & 1 & 2 & 2 & 1 & 2 & 1 & 1 & 2 & 1 & 2 & 2 & 1 & 2 & 1 \\
\hline 12 & 2 & 1 & 2 & 2 & 1 & 2 & 1 & 2 & 1 & 2 & 1 & 1 & 2 & 1 & 2 \\
\hline 13 & 2 & 2 & 1 & 1 & 2 & 2 & 1 & 1 & 2 & 2 & 1 & 1 & 2 & 2 & 1 \\
\hline 14 & 2 & 2 & 1 & 1 & 2 & 2 & 1 & 2 & 1 & 1 & 2 & 2 & 1 & 1 & 2 \\
\hline 15 & 2 & 2 & 1 & 2 & 1 & 1 & 2 & 1 & 2 & 2 & 1 & 2 & 1 & 1 & 2 \\
\hline 16 & 2 & 2 & 1 & 2 & 1 & 1 & 2 & 2 & 1 & 1 & 2 & 1 & 2 & 2 & 1 \\
\hline
\end{tabular}

$A I=$ sum of the outputs when $A$ is low

$A 2=$ sum of the outputs when $A$ is high

Effect of $A=|A 1-A 2|$

Table 3. Effects of design parameters

\begin{tabular}{|c|c|c|c|c|c|}
\hline Var & Load 1 & Load 2 & Load 3 & Load 4 & Average \\
\hline $\mathbf{t 4}$ & 0.59 & 1.09 & 1.49 & 1.81 & 1.25 \\
\hline $\mathbf{E}$ & 0.36 & 1.02 & 1.42 & 1.77 & 1.14 \\
\hline $\mathbf{t 3}$ & 0.41 & 0.78 & 1.10 & 1.38 & 0.92 \\
\hline $\mathbf{t 2}$ & 0.48 & 0.83 & 1.07 & 1.22 & 0.90 \\
\hline $\mathbf{G}$ & 0.32 & 0.59 & 0.81 & 0.98 & 0.67 \\
\hline $\mathbf{H}$ & 0.22 & 0.40 & 0.53 & 0.64 & 0.45 \\
\hline $\mathbf{r 2}$ & 0.15 & 0.32 & 0.50 & 0.67 & 0.41 \\
\hline $\mathbf{f 2}$ & 0.11 & 0.19 & 0.25 & 0.47 & 0.26 \\
\hline $\mathbf{f 4}$ & 0.11 & 0.18 & 0.21 & 0.24 & 0.18 \\
\hline $\mathbf{f 3}$ & 0.07 & 0.14 & 0.20 & 0.25 & 0.17 \\
\hline $\mathbf{W}$ & 0.06 & 0.13 & 0.20 & 0.27 & 0.17 \\
\hline $\mathbf{f 1}$ & 0.07 & 0.13 & 0.16 & 0.18 & 0.14 \\
\hline BT & 0.05 & 0.08 & 0.10 & 0.11 & 0.08 \\
\hline TT & 0.03 & 0.04 & 0.05 & 0.05 & 0.04 \\
\hline r1 & 0.03 & 0.04 & 0.03 & 0.02 & 0.03 \\
\hline
\end{tabular}


The significance values provide insight into the amount of influence that any one parameter has on the response of the pad. From the above table, it is clear that the thickness of the oval beam member $\left(\mathrm{t}_{4}\right)$ in the unit cell exerts the greatest influence on the system, both for stiffening and softening, depending on if it's set at the high or low level. These values for the parameters will prove to be useful in future design challenges, and provide information on which elements of the unit cell should garner the most attention if redesigns and adjustments are needed for this particular design problem. Another interesting takeaway from these significance values is the remarkable effect that essential support geometry (ESG) thicknesses have compared to the functional geometry (EFG) parameters. The ESG thickness (t3) rank third in the table of effect coefficients, while the primary function of the ESG is to provide support and allow the UCs to be tessellated in an offset pattern.

\subsubsection{Interaction plots}

In our effort to best match the experimental test, the softest allowable pad given the maximum printing inaccuracies needed to be found. While individual parametric contributions towards mechanical behavior are of significant worth, what is arguably more important is ascertaining how interactions between separate parametric changes compound in the mechanical behavior. It is also essential to know if there is a combination of increases and decreases that generates a more optimal response than blanket parameter increases or decreases. Therefore, a study of interactions was completed as a result of the DOE. By observing interaction plots, an example of which is shown in Figure 6, the strength of a particular interaction between two variables can be observed. The parallelism of two lines in a plot indicates the magnitude of interaction, with parallel lines indicating no interaction and intersecting lines indicating a strong interaction. Everything in between indicates some level of weaker interaction.

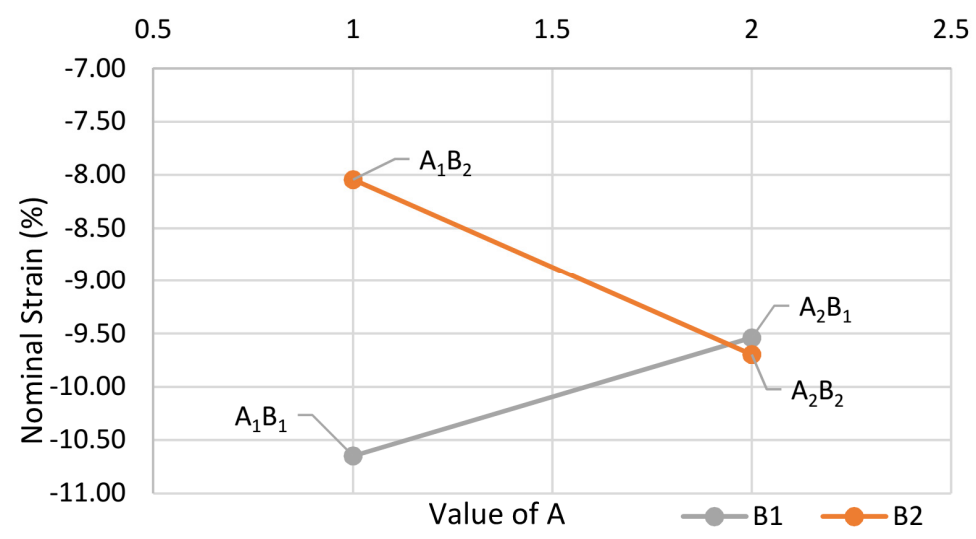

Figure 6. UC half width $x$ CB thickness interaction plot

For the plot in Figure 6, a strong interaction was observed between the width of the unit cell (W) and the thickness of the cantilever beam ( $\mathrm{t} 2$ ). The plot shows that changing both of these values produces a greater overall effect than independent effects added together. Additionally, the plot shows higher strains for all experiments with an $\mathrm{A}_{2} \mathrm{~B}_{2}$ combination, as compared to the experiments with an $\mathrm{A}_{2} \mathrm{~B}_{1}$ combination. In this case, 2 indicates a high value and 1 indicates a low value. This means that for all the experiments where $\mathrm{CB}$ thickness was set to the upper bound, the softest responses came when $\mathrm{W}$ was also set to the upper bound. When we look at the construction procedure of the UC, this result can be corroborated using EulerBernoulli beam theory. As the width of the unit cell is increased, the cantilever beam member is lengthened, placing the force acting on the cell farther away from the fixed end of the beam. This results in increased displacements of the beam tip, which correlates to increases in overall pad deformation across the entirety of the profile. If beam lengthening is coupled with a decrease in thickness, and the subsequent moment of inertia, the resultant displacement as a result of the force will be even greater.

Through the analysis of other plots, significant interactions were observed between the various member thicknesses and the height and width of the cells. Further investigation, for example, showed that the $\mathrm{H}$ of the unit cell and the thickness of the ESG members interacted strongly with one another. This allowed us to build a much more complete understanding of the geometrical relationships present in the pad, 
showing which parameters to increase and which parameters to decrease in order to model the softest possible pad within known printing tolerances. Ultimately, an increase in both $\mathrm{H}$ and $\mathrm{W}$, coupled with decreases in the remaining variables proved to provide the softest pad behavior that could be reasonably expected from the EBM process. Figure 7 shows the response of this particular dimensional combination compared to the original target and experimental responses and the changes are seen to account for more than $25 \%$ of the difference between the target and real curves. While it is unreasonable to expect all the dimensions to rest at the appropriate upper and lower bounds to produce this specific scenario, the figure shows the discernible effect inaccuracies could have on the performance of the pad and other geometrically intricate parts.

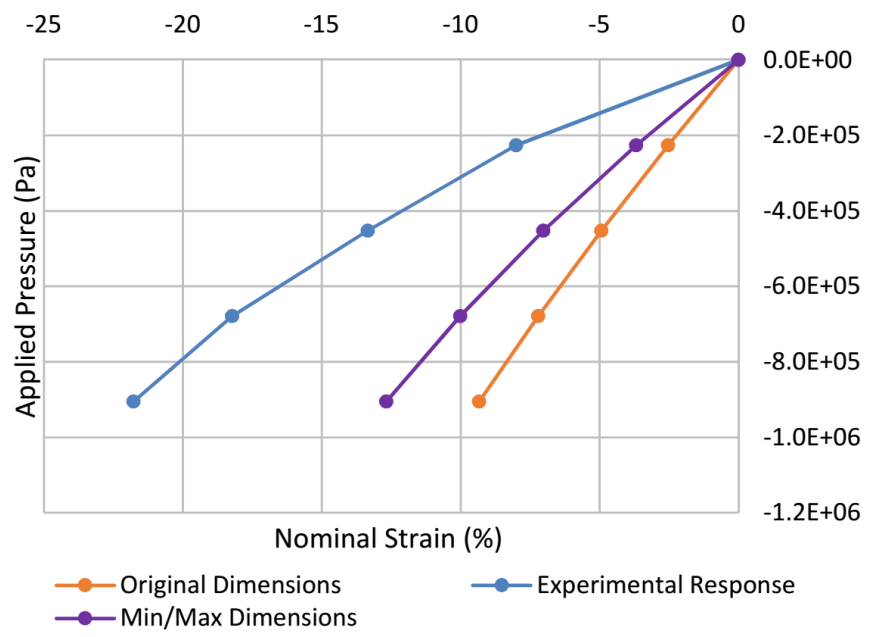

Figure 7. Effects of combined potential dimensional inaccuracies

\subsection{Boundary conditions}

Being one of the three influential factors which could explain the obtained results, there is a belief that the boundary conditions will contribute in a significant manner to the results produced by the model. Preliminary work has been conducted to most accurately represent the experimental setup and associated boundary conditions. While initial results do show larger strains for the model, as shown in Figure 8, loss of nonlinearity in the deformations was unexpected and contrary to the corresponding experimental results. Therefore, more research regarding the optimal ways to represent the contact between the moving parts of the experiment is needed. Other factors must also be identified, such as the coefficients of friction between the pad and plates and the most appropriate ways to assign boundary conditions and constraints to all the pieces.

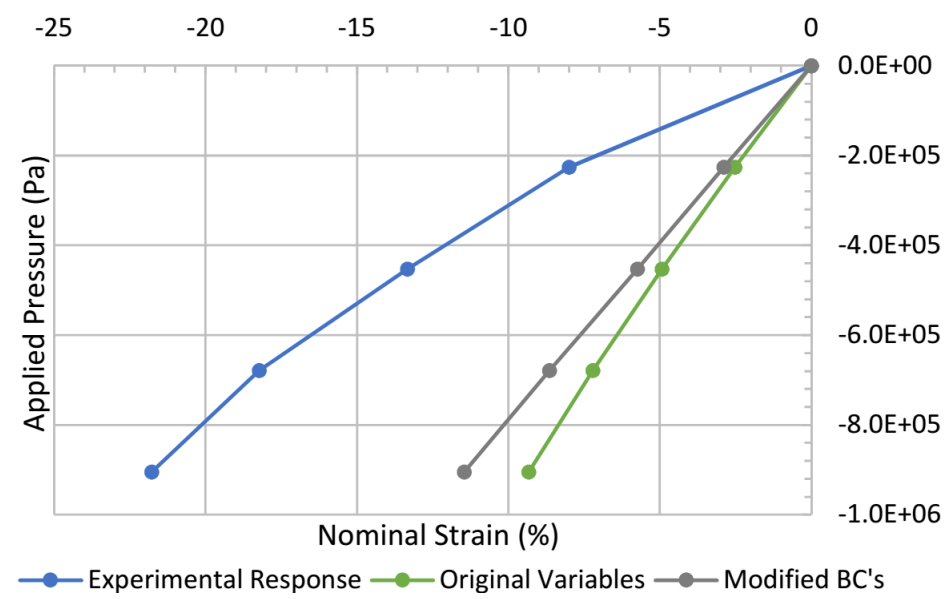

Figure 8. Effects of improved boundary conditions 


\section{Conclusions and future work}

As a result of this investigation, several significant takeaways emerged that could help influence the material design community with more time and work invested. Even though the experiment did not produce results that were expected, the pad did behave nonlinearly as intended despite being manufactured from a linear elastic material. This result provides a level of validation to the design model. Another conclusive result from this investigation is the importance of printing accuracy in EBM and other additive manufacturing processes. While EBM boasts an impressive tolerance of $0.1 \mathrm{~mm}$, other printing methods have even higher margins of error. The sensitivity analysis of the manufactured pad shows that, in certain combinations, the potential inaccuracies could drastically alter the expected performance of a part. The ability for two or more dimensional changes to magnify the overall negative response is also an important detail to carry forward into future designs, and finding ways to identify and mitigate these compounding effects should be high on the list of future work. These possibilities could push more serious considerations of robustness and reliability into cellular material design, as the capabilities of AM continue to be called upon to address new and novel goals. The same can be said for the importance of consistent material properties, as the results showed that changes across the known spectrum of stiffness for Ti-6Al-4V can single-handedly disrupt the desired mechanical behavior of the product.

Lastly, recommendations for a more pre-emptive implementation of these studies can be made to designers of cellular materials, and designers for additive manufacturing as a whole. While this particular study was conducted as a search for causation, utilizing sensitivity analyses to bridge the gap between the final design selection and part manufacturing could provide an opportunity to add an additional layer of robustness and reliability to processes like the MUCSM. With these designs utilizing optimized features only a magnitude of $10^{1}$ or $10^{2}$ higher than the tolerance threshold, analyzing a unit cell's receptiveness to parameter change can provide insight into what features should be used given considerations and manufacturing limitations. Additionally, new features could be added to the design arsenal, which are more resistive to build errors or the adverse effects of feature combinations. All of this would allow designers to make more informed and reliable decisions in current and subsequent unit cell designs and provide products that are more consistently in line with their original objectives.

Future work needs to be done. Primarily, more pads are needed to obtain a more representative sample size of EBM printed parts. With only one data point, it is impossible to conclusively state what the causes for the discrepancy are, or if the current test data is even accurate. Accompanying the production of more pads, having tensile and compression test specimens printed would prove invaluable, allowing for independent confirmation of mechanical properties for EBM parts. It would also provide an opportunity to alter the printing orientation of the specimens, thus providing a more exhaustive understanding of how highly optimized parts should be printed to obtain the most conducive mechanical and material properties.

\section{Acknowledgements}

This project was funded by the Automotive Research Center, a U.S. Army center of excellence for modeling and simulation of ground vehicles, led by the University of Michigan College of Engineering. The views presented here do not necessarily reflect those of our sponsor, who's support is gratefully appreciated.

\section{References}

Alharbi, N., Osman, R. and Wismeijer, D. (2016), "Factors Influencing the Dimensional Accuracy of 3D-Printed Full-Coverage Dental Restorations Using Stereolithography Technology", The International Journal of Prosthodontics, Vol. 29 No. 5, pp. 503-510. https://doi.org/10.11607/ijp.4835

Cooke, A.L. and Soons J.A. (2010), "Variability in the geometric accuracy of additively manufactured test parts", The 21st Annual Solid Freeform Fabrication Symposium: An Additive Manufacturing Conference, Austin, TX, USA, August 9-11, 2010, pp. 1-12.

Cui, T.J., Smith, D. and Liu, R. (2010), Metamaterials: Theory, Design, and Applications, Springer. https://doi.org/10.1007/978-1-4419-0573-4

Dimitrov, D., van Wijck, W., Schreve, K., and de Beer, N. (2006), "Investigating the achievable accuracy of three dimensional printing", Rapid Prototyping Journal, Vol. 12 No. 1, pp. 42-52. https://doi.org/10.1108/13552540610637264 
Farzadi, A., Solati-Hashjin, M., Asadi-Eydivand, M. and Abu Osman, N.A. (2014), "Effect of Layer Thickness and Printing Orientation on Mechanical Properties and Dimensional Accuracy of 3D Printed Porous Samples for Bone Tissue Engineering", PLoS ONE, Vol. 9 No. 9, pp. e108252. https://doi.org/10.1371/journal.pone.0108252

Gu, S., Lu, T.J. and Evans, A.G. (2001), "On the design of two-dimensional cellular metals for combined heat dissipation and structural load capacity", International Journal of Heat and Mass Transfer, Vol. 44 No. 11, pp. 2163-2175. https://doi.org/10.1016/s0017-9310(00)00234-9

Hernandez, D.D. (2015), "Factors Affecting Dimensional Precision of Consumer 3D Printing", International Journal of Aviation, Aeronautics, and Aerospace, Vol. 2 No. 4. https://doi.org/10.15394/ijaaa.2015.1085

Ju, J., Ananthasayanam, B., Summers, J. and Joseph, P. (2010), "Design of Cellular Shear Bands of a NonPneumatic Tire -Investigation of Contact Pressure", SAE International Journal of Passenger Cars Mechanical Systems, Vol. 3 No. 1, pp. 598-606. https://doi.org/10.4271/2010-01-0768

Kulkarni, N.M. (2016), Design Optimization of Tank Track Pad Meta-Material Using the Cell Synthesis Method, Master thesis, Clemenson University.

Levy, G., Spierings, A.B. and Wegener, K. (2012), "Designing Material Properties Locally with Additive Manufacturing technology SLM”, SFF Symposium, Austin, USA, pp. 447-455.

Lieneke, T., Adam, G.A.O., Leuders, S., Knoop, F., Josupeit, S. et al. (2015), "Systematical Determination of Tolerances for Additive Manufacturing by Measuring Linear Dimensions", SFF Symposium, Austin, USA, pp. 371-384.

Rosen, D., Johnston, S., Reed, M. and Wang, H. (2006), "Design of General Lattice Structures for Lightweight and Compliance Applications", Rapid Manufacturing Conference 2006.

Ruzzene, M. (2003), "Vibration and Sound Radiation of Sandwich Beams with Honeycomb Truss Core", Noise Control and Acoustics, Washington, DC, USA, November 15-21, 2003, ASME, pp. 1-14. https://doi.org/10.1115/imece2003-41552

Safdar, A., He, H.Z., Wei, L., Snis, A. and Chavez de Paz, L.E. (2012), "Effect of process parameters settings and thickness on surface roughness of EBM produced Ti-6Al-4V", Rapid Prototyping Journal, Vol. 18 No. 5, pp. 401-408. https://doi.org/10.1108/13552541211250391

Salmi, M., Paloheimo, K.-S., Tuomi, J., Wolff, J. and Mäkitie, A. (2013), “Accuracy of medical models made by additive manufacturing (rapid manufacturing)", Journal of Cranio-Maxillofacial Surgery, Vol. 41 No. 7, pp. 603-609. https://doi.org/10.1016/j.jcms.2012.11.041

Satterfield, Z., Kulkarni, N., Fadel, G., Li, G., Coutris, N. and Castanier, M.P. (2017), "Unit Cell Synthesis for Design of Materials with Targeted Nonlinear Deformation Response", Journal of Mechanical Design, Vol. 139 No. 12, pp. 121401. https:/doi.org/10.1115/1.4037894

Schade, C.T., Murphy, T.F. and Walton, C. (2014), "Development of atomized powders for additive manufacturing", World Congress on Powder Metallurgy and Particulate Materials (PM 2014), Orlando, USA, May 18-22, 2014, pp. 215-226.

Schultz, J., Griese, D., Ju, J., Shankar, P., Summers, J.D. and Thompson, L. (2012), "Design of Honeycomb Mesostructures for Crushing Energy Absorption”, Journal of Mechanical Design, Vol. 134 No. 7, pp. 071004. https://doi.org/10.1115/1.4006739

Smith, C.J., Derguti, F., Hernandez Nava, E., Thomas, M., Tammas-Williams, S. et al. (2016), "Dimensional accuracy of Electron Beam Melting (EBM) additive manufacture with regard to weight optimized truss structures", Journal of Materials Processing Technology, Vol. 229, pp. 128-138. https://doi.org/10.1016/j.jmatprotec.2015.08.028

Spierings, A.B., Voegtlin, M., Bauer, T. and Wegener, K. (2015), "Powder flowability characterisation methodology for powder-bed- based metal additive manufacturing", Progress in Additive Manufacturing, Vol. 1 No. 1-2, pp. 9-20. https://doi.org/10.1007/s40964-015-0001-4

Spierings, A.B., Herres, N. and Levy, G. (2011), "Influence of the particle size distribution on surface quality and mechanical properties in AM steel parts", Rapid Prototyping Journal, Vol. 17 No. 3, pp. $195-202$. https://doi.org/10.1108/13552541111124770

Strondl, A., Lyckfeldt, O., Brodin, H. and Ackelid, U. (2015), "Characterization and Control of Powder Properties for Additive Manufacturing”. JOM, Vol. 67 No. 3, pp. 549-554. https://doi.org/10.1007/s11837015-1304-0

Thyagaraja, N., Shankar, P., Fadel, G. and Guarneri, P. (2011), "Optimizing the Shear Beam of a Non-Pneumatic Wheel for Low Rolling Resistance", Volume 5: 37th Design Automation Conference, Parts $A$ and B, Washington, DC, USA, August 28-31, 2011, ASME, pp. 33-42. https:/doi.org/10.1115/detc2011-48532

Varanasi, S., Bolton, S.J., Siegmund, T.H. and Cipra, R.J. (2013), "The low frequency performance of metamaterial barriers based on cellular structures", Applied Acoustics, Vol. 74 No. 4, pp. 485-495. https://doi.org/10.1016/j.apacoust.2012.09.008 
Wadley, H.N.G. and Queheillalt, D.T. (2007), “Thermal Applications of Cellular Lattice Structures”, Materials Science Forum, Vol. 539, pp. 242-247.

Wang, H.V. (2005), A Unit Cell Approach for Lightweight Structure and Compliant Mechanism, PhD thesis, Georgia Tech.

Prof. Georges Fadel

Clemson University, Mechanical Engineering

202 Fluor Daniel EIB, 29634-0921 Clemson, United States

Email: fgeorge@clemson.edu 